\title{
TCEC14: the 14th top chess engine championship
}

Article

Accepted Version

The official report

Haworth, G. and Hernandez, N. (2019) TCEC14: the 14th top chess engine championship. ICGA Journal, 41 (3). pp. 143151. ISSN 1389-6911 doi: https://doi.org/10.3233/ICG-190117 Available at https://centaur.reading.ac.uk/82052/

It is advisable to refer to the publisher's version if you intend to cite from the work. See Guidance on citing.

Published version at: https://content.iospress.com/articles/icga-journal/icg190117

To link to this article DOI: http://dx.doi.org/10.3233/ICG-190117

Publisher: The International Computer Games Association

All outputs in CentAUR are protected by Intellectual Property Rights law, including copyright law. Copyright and IPR is retained by the creators or other copyright holders. Terms and conditions for use of this material are defined in the End User Agreement.

\section{www.reading.ac.uk/centaur}

\section{CentAUR}

Central Archive at the University of Reading

Reading's research outputs online 


\section{TCEC14: the $14^{\text {th }}$ Top Chess Engine Championship}

Guy Haworth and Nelson Hernandez ${ }^{1}$

Reading, UK and Maryland, USA

TCEC Season 14 started on November $12^{\text {th }} 2018$ and introduced a number of changes from TCEC 13 (Haworth and Hernandez, 2019b). An enlarged Division 4 featured twelve engines and seven newcomers to accommodate the increasing interest in computer chess and this competition in particular. The other divisions remained eight strong. The five divisions played two or more double round-robins ('DRR') each with promotions and relegations following. Tempi gradually lengthened from 'Rapid' to 'Classical', and the Premier division's top two engines played a 100-game match to determine the Grand Champion.

The trio of STOCKFISH, KOMODO and HOUDINI have dominated the TCEC medals for several seasons and a key point of interest was whether others would reach the podium. LEELA CHESS ZERO and ETHEREAL were certainly expected to perform well in Division P, having shown remarkable improvement in the previous few months. KOMODO MCTS was a dark horse.

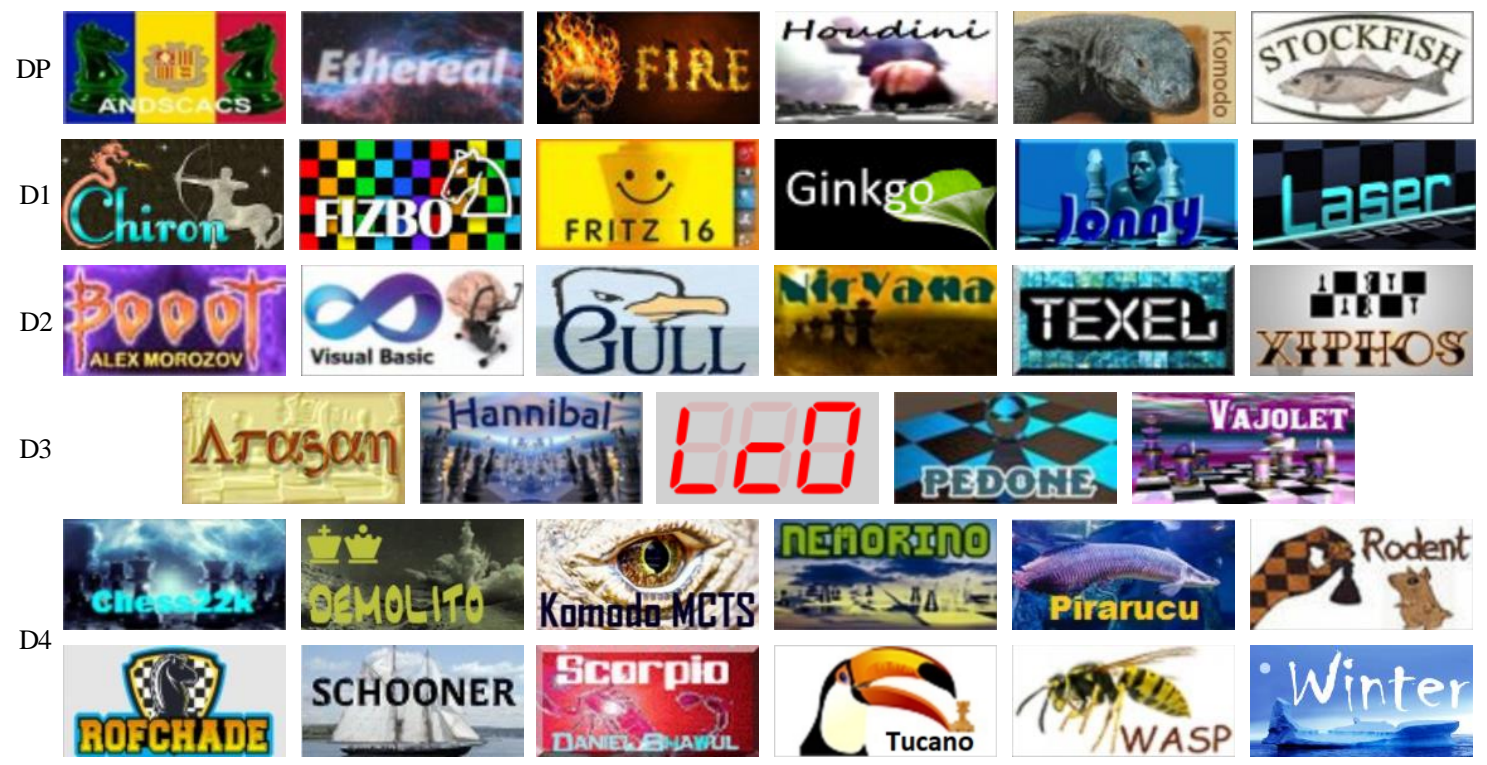

Fig. 1. Logos for the TCEC 14 engines (CPW, 2019) as in their original divisions.

There were a few nudges to TCEC's adjudication rules. Draw adjudication could be invoked after move 35 (rather than move 40) and the two engines had to both evaluate within \pm 0.08 (rather than \pm 0.05 ) for

\footnotetext{
${ }^{1}$ Corresponding author: g.haworth@ reading.ac.uk
} 
eight consecutive plies and with plycount $\neq 0$. While draw-adjudication requirements were relaxed, win-adjudication requirements were tightened. Engine evaluations had to be outside \pm 10 (rather than \pm 6.5 ) for ten consecutive plies (rather than eight); plycount was not a factor. This change was welcomed by those of us who wanted to see a clearer demonstration of superiority on the board: it will be interesting to see how long it prolongs the decisive games and what mysteries remain.

The common platform for TCEC14 consisted of two computers. One was the established, formidable 44-core server of TCEC11-13 (Intel, 2017) with 64GB of DDR4 ECC RAM and a Crucial CT250M500 240 GB SSD for the EGTs. The 'GPU server', a Quad Core i5 2600k, was sporting Nvidia (2018) GeForce RTX 2080 Ti and 2080 GPUs for those engines which could exploit them.

\section{The engines}

Season 13 competitors BoBCAT, DEUS X, HANNIBAL and SENPAI rested for this TCEC season. TCEC welcomed first appearances for engines DEMOLITO, KOMODO MCTS, PIRARUCU, ROFCHADE, SCHOONER, SCORPIONN and WINTER, see Fig. 1 and Table 1.

Table 1. The TCEC14 engines (CP, 2019), details, authors and progress.

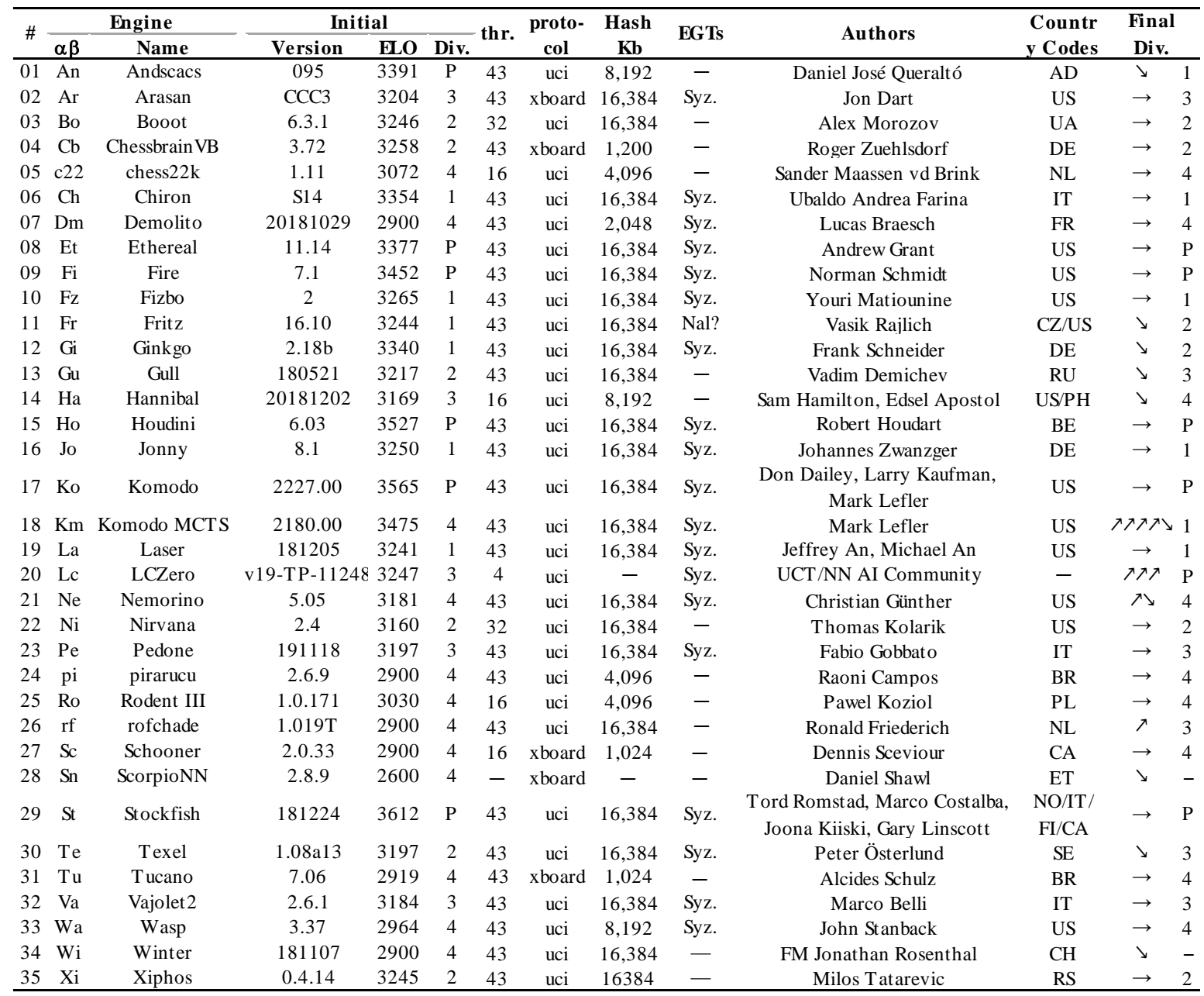




\section{Division 4: 2 DRR Phases, 4 round robins, 264 games, 30' $+10^{\prime \prime} / \mathrm{m}$}

As for TCEC12/13, each engine played both White and Black from four-ply openings defined by the second author here. The results are as in Table 2: 'P\%' is the \%-score and ' $\mathrm{ELO} \pm$ ' is the change to the engine's nominal ELO based on its performance. Generic stats are in Tables 9 and 10.

Online interest naturally focused on the newcomers, especially KOMODO MCTS (Chessdom, 2018), a further innovation from the Lefler/Kaufman camp. The engines had a wide range of ability leading to only $34.1 \%$ of games being drawn: those given a default 'TCEC-entry ELO' of 2900 ranged across the field. WINTER was always headed for a demotion spot. SCORPIONN clearly was not ready for the contest and even though it disconnected eight times, it did not impact the ranking elsewhere. The bottom three missed TCEC Cup 2 (Haworth and Hernandez, 2019c). The three engines promoted were clearly ahead: KOMODO MCTS, ROFCHADE and NEMORINO.

Table 2. The TCEC14 Division 4 cross-table: two DRR phases, 44 rounds, 264 games.

\begin{tabular}{|c|c|c|c|c|c|c|c|c|c|c|c|c|c|c|c|c|c|c|}
\hline \# & & ELO & Pts & P\% & Elo \pm & SB & $\mathbf{K m}$ & rf & $\mathrm{Ne}$ & Sc & pi & Wa & Dm & Tu & Ro & c22 & Wi & Sn \\
\hline 01 & Komo & 3475 & 37.5 & 85.2 & -133 & 745.75 & & $==01$ & $111=$ & $11==$ & 1111 & 1111 & $11=1$ & $=11=$ & $=111$ & $1=11$ & $111=$ & 1111 \\
\hline & & 2900 & 2.5 & 73.9 & +461 & 627.75 & & & & $====$ & & & & & & & & 1111 \\
\hline & & & & 68.2 & -96 & & & & & $===1$ & & & & & & & & $: 1111$ \\
\hline & & & 8.0 & 63.6 & & & & $==$ & $===0$ & & & & & & & & & 1111 \\
\hline & & & & & 05 & & & $=01$ & & $=10=0$ & & & & & & & & $=111$ \\
\hline & & & & .7 & & & & $==0=$ & & $====$ & & & & & & & & 111 \\
\hline 07 & & 2900 & 23.0 & 52.3 & +157 & 409.50 & & $00=0$ & & $=1=$ & 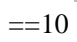 & & & $0=11$ & & & & 1111 \\
\hline 08 & & & & 40.9 & & & & $=000$ & & $0===$ & $0===$ & & $1=00$ & & & & & 1111 \\
\hline 09 & & 3030 & 7.5 & 39.8 & 56 & 66.50 & & 0000 & $=0$ & 0000 & $0===$ & $=0=0$ & $1==0$ & $10==$ & & $==10$ & $=111$ & 1111 \\
\hline 10 & & 307 & & 38.6 & -344 & 279.75 & & $0===$ & 00 & $=000$ & $0==0$ & 00 & $===0$ & $10=0$ & $==01$ & & $1==1$ & 1111 \\
\hline & & & & 25.0 & & 0 & & & & $000=$ & & & & & & $==0$ & & $1=11$ \\
\hline 12 & & 2600 & 0.5 & 01.1 & -135 & 5.50 & 0000 & 0000 & & 0000 & 0000 & & 0000 & & 0000 & 0000 & & \\
\hline
\end{tabular}

\section{Division 3: two DRR phases, 14 rounds, 112 games, tempo $30^{\prime}+10^{\prime \prime} / \mathrm{m}$}

Again, the eight engines involved played both sides of 14 prescribed four-ply openings. With GPU operating temperatures more stable, LCZERO was expected to do well after its performance in TCEC Cup 1 (Haworth and Hernandez, 2019a) and it did not disappoint, see Table 3. KoMODO MCTS also distanced the rest of the field and continued on up the divisions. Crashes remained a problem: this time, HANNIBAL incurred five. ${ }^{2}$ In game 26/7.2, ROFCHADE disconnected in the KBPKBPP position 95b, a 7-man tablebase draw.

Table 3. The TCEC14 Division 3 cross-table: two DRR phases, 28 rounds, 112 games.

\begin{tabular}{|c|c|c|c|c|c|c|c|c|c|c|c|c|c|c|}
\hline \# & Engine & ELO & Pts & $\mathbf{P \%}$ & Elo \pm & S B & Lc & $\mathbf{K m}$ & Pe & Ar & Va & rf & $\mathrm{Ne}$ & Ha \\
\hline 1 & LCZero v19-TP-11248 & 3247 & 22.5 & 80.4 & +310 & 283.00 & & $=1=1$ & $11==$ & $0=11$ & 1111 & $=1=1$ & $11=1$ & $=111$ \\
\hline 2 & Komodo MCTS 2210.00 & 3479 & 20.0 & 71.4 & -60 & 238.00 & $=0=0$ & & $11=1$ & $11==$ & $=11=$ & $=1=1$ & $1=$ & 1111 \\
\hline 3 & Pedone 191118 & 3197 & 13.5 & 48.2 & +88 & 158.75 & $00==$ & $00=0$ & & $1==0$ & $=0=0$ & $===1$ & 1110 & $1=11$ \\
\hline 4 & Arasan CCC3 & 3204 & 13.5 & 48.2 & +79 & 171.75 & $1=00$ & $00==$ & $0==1$ & & $====$ & $10==$ & $====$ & $1==1$ \\
\hline 5 & Vajolet2 2.6.1 & 3184 & 12.5 & 44.6 & +73 & 151.00 & 0000 & $=00=$ & $=1=1$ & $====$ & & $=011$ & $=0==$ & $==1=$ \\
\hline 6 & rofChade $1.023 \mathrm{~T}$ & 3376 & 12.5 & 44.6 & -181 & 158.00 & $=0=0$ & $=0=0$ & $===0$ & $01==$ & $=100$ & & $==11$ & $=1==$ \\
\hline 7 & Nemorino 5.05 & 3360 & 11.5 & 41.1 & -193 & 143.50 & $00=0$ & $=0==$ & 0001 & $====$ & $=1==$ & $==00$ & & 1011 \\
\hline 8 & Hannibal 20181202 & 3169 & 6.0 & 21.4 & -116 & 80.50 & $=000$ & 0000 & $0=00$ & $0==0$ & $==0=$ & $=0==$ & 0100 & \\
\hline
\end{tabular}

\footnotetext{
${ }^{2}$ It sat out TCEC15 while the engineers looked under the bonnet.
} 


\section{Division 2: two DRR phases, 14 rounds, 112 games, tempo $30^{\prime}+10^{\prime \prime} / \mathrm{m}$}

Game 64/16.4, KOMODOMCTS-LEELA, ended in a rare stalemate on m172. Game 93/24.1, NIRVANALEELA, was a KBNPKRN draw at positions 115b and 165w: NIRVANA claimed the draw with 165. Bd6 - which loses to $165 . . . \mathrm{Nf7}+166 . \mathrm{K} \sim \mathrm{Nxd6}$. Do chess programs do irony? Demoted GULL beat BooOT and BOOOT beat LEELA which otherwise moved smoothly away to win the division again, see Table 4 . KOMODO MCTS took silver with one less loss to LEELA than XIPHOS and one more win elsewhere.

Table 4. The TCEC14 Division 2 cross-table: two DRR phases, 28 rounds, 112 games.

\begin{tabular}{|c|c|c|c|c|c|c|c|c|c|c|c|c|c|c|}
\hline \# & Engine & ELO & Pts & $\mathbf{P \%}$ & Elo \pm & SB & $\mathbf{L c}$ & $\mathbf{K m}$ & $\mathbf{X i}$ & Bo & $\mathbf{N i}$ & $\mathbf{C b}$ & Gu & Te \\
\hline 1 & LCZero v19.1-RC2-11248 & 3247 & 20.0 & 71 & +200 & 260.50 & & $=1==$ & $=11=$ & $110=$ & $1=1=$ & $1=1=$ & $=11=$ & $1=1=$ \\
\hline 2 & Komodo MCTS 2217.00 & 3479 & 17.0 & 61 & -182 & 216.75 & $=0==$ & & $==$ & $===$ & $1===$ & $=1==$ & $1==1$ & $11=1$ \\
\hline 3 & Xiphos 0.4.14 & 3245 & 16.0 & 57 & +75 & 207.00 & $=00=$ & $====$ & & $====$ & $11==$ & $==11$ & $===$ & $===1$ \\
\hline 4 & Booot 6.3.1 & 3246 & 14.0 & 50 & +10 & 188.50 & $001=$ & $====$ & $====$ & & $====$ & $0===$ & $0=1=$ & $=1=1$ \\
\hline 5 & Nirvana 2.4 & 3160 & 13.0 & 46 & +96 & 169.25 & $0=0=$ & $0===$ & $00=-$ & $====$ & & $01==$ & $=11$ & $==1=$ \\
\hline 6 & ChessBrainVB 3.72 & 3258 & 12.0 & 43 & -71 & 164.00 & $0=0=$ & $=0==$ & $==00$ & $1===$ & $10==$ & & $11=0$ & $==0=$ \\
\hline 7 & Gull 180521 & 3217 & 11.5 & 41 & -30 & 149.75 & $=00=$ & $0==0$ & $===0$ & $1=0=$ & $=00=$ & $00=1$ & & $111=$ \\
\hline 8 & Texel 1.08a13 & 3197 & 8.5 & 30 & -98 & 121.75 & $0=0=$ & $00=0$ & $===0$ & $=0=0$ & $==0=$ & $==1=$ & $000=$ & \\
\hline
\end{tabular}

\section{Division 1: two DRR phases, 28 rounds, 112 games, tempo $60^{\prime}+10^{\prime \prime} / \mathrm{m}$}

The penultimate game 28.3/111 was the longest ever for TCEC Division 1 at 308 moves: 'new wave' LEELA versus 'old guard, oldest brand' FRITZ 16. The win is routine enough with rook and passed pawn against a half-sighted bishop but endgame solver FINALGEN (Romero, 2012) sees 20 moves before a clear win, a line that results in mate on move 337 at best (Haworth and Hernandez, 2019d).

GINKGO surprisingly crashed four times and was disqualified so the formal results are slightly different from those of Table 5 even if promotions/relegations are otherwise unaffected. FRITZ never saw a win in this company and also was demoted to division 2.

Table 5. The TCEC14 Division 1 cross-table: two DRR phases, 28 rounds, 112 games.

\begin{tabular}{|c|c|c|c|c|c|c|c|c|c|c|c|c|c|c|}
\hline \# & Engine & ELO & Pts & P\% & Elo \pm & SB & Lc & Km & $\mathbf{F z}$ & Ch & Gi & $\mathbf{L a}$ & Jo & Fr \\
\hline 1 & LCZero v19.1-TC2-11248 & 3227 & 19.5 & 70 & 231 & 253.75 & & & $=11$ & $11=0$ & $==11$ & $1=11$ & $==1$ & 1101 \\
\hline 2 & ICT C 220100 & 3200 & & 63 & 204 & 230.25 & $====$ & & $==10$ & $=$ & & $===$ & $===1$ & $11==$ \\
\hline 3 & & 3286 & & 52 & & 195.00 & $==00$ & $==01$ & & $1===$ & & $==10$ & $==1$ & $====$ \\
\hline 4 & Chi & 3379 & 13.5 & 48 & -172 & 177.25 & $00=1$ & $=0==$ & $0===$ & & $10=0$ & $===$ & $=10=$ & $1=11$ \\
\hline 5 & Gink & 3365 & 13.5 & 48 & -154 & 175.75 & $==00$ & $==00$ & $001=$ & $01=1$ & & $====$ & $1==1$ & $=1==$ \\
\hline 6 & Lase & 3216 & 13.0 & 46 & +38 & 170.25 & $0=00$ & $===0$ & $==01$ & $====$ & $==$ & & $=1==$ & $1===$ \\
\hline 7 & Jonn & 3256 & 12.0 & 43 & -50 & 162.75 & $==0=$ & $===($ & $==0$ & $=01=$ & $0==0$ & $=0==$ & & $=1=1$ \\
\hline 8 & .10 & 3200 & 3.5 & 30 & -84 & & & & & & & & & \\
\hline
\end{tabular}

\section{Division $P$, three DRR phases, 42 rounds, 168 games, tempo $90^{\prime}+10^{\prime \prime} / \mathrm{m}$}

The line-up for Division P had only a semi-familiar look. After the TCEC13 podium trio of STOCKFISH, KOMODO and HOUDINI, we had the other survivors FIRE, ETHEREAL and ANDSCACS. Interest however centred on the newcomers LEELA CHESS ZERO and KOMODO MCTS, both bringing MCTS search to the game. The contest was three DRRs rather than the four of TCEC13. 
After the first round-robin, STOCKFISH had jumped out into the lead with four wins. After the first DRR, with colour-bias eliminated, STOCKFISH maintained a healthy lead and remained unbeaten, a feat shared with KOMODO and LEELA. Was the TCEC podium about to change? KOMODO MCTS had disconnected and lost twice against KOMODO in drawn positions. A third disconnection would be bad for both engines: disqualification for MCTS and elimination of Komodo's crash-wins from the table.

Game 64 saw STOCKFISH beat KoMODO, opening the door for LEELA. In game 68 at the foot of the table, ANDSCACS beat ETHEREAL with Black. At the half-way point, LEELA was edging the contest for second place and remained unbeaten. The fourth round-robin saw LEELA consolidate its second place with four straight wins against the tail including one as Black against ETHEREAL. The competition for second place remained open as STOCKFISH finally ended LEELA's unbeaten run in the last RR4 game, g28.4/112.

The fifth round-robin saw plenty of drama. LEELA lost as Black to both KOMODO and FIRE, the first having serious tie-break significance and the second being seriously unexpected. GPU fan-settings were thought to be a contributory factor but not enough to trigger replays. In game 33.1/129 v HouDINI, KOMODO MCTS disconnected for a third time, was disqualified and relegated with its games discounted. Hopefully, Mark Lefler will sort out the technical problems for TCEC15. This restored LEELA to second place. With one round-robin to go, adjusted scores at the top were STOCKFISH well clear on 21, LEELA 16.5, KOMODO and HOUDINI 16. The second relegation spot was between ETHEREAL on 11.5 and ANDSCACS on 11.

Every win was now going to be a major event, especially as the last round of 28 games started with seven draws. KOMODO as White lost to STOCKFISH in g37.4/148. Both LEELA and KOMODO beat FIRE. In the penultimate game, KOMODO beat ANDSCACS: ETHEREAL breathed again, having narrowly survived without a single win in this division. In the last game, a cliffhanger, STOCKFISH searched the endgame tables a thousand times more than LEELA and thought it had a feasible advantage, but LEELA held out in KRPPKRP to draw on move 93.

The raw figures of Tables 6 and 7 need adjustment because KOMODO MCTS' disqualification flipped the ranking at both ends of the table. In fact, STOCKFISH ultimately had 25 points, LEELA 20, KOMODO 19.5, ETHEREAL 14 and ANDSCACS 13.5. The 'big three' became the 'big four' but the Shannon-AB engine mould was cracked again: the still-improving LEELA had remarkably progressed from Division 3 all the way to the TCEC Superfinal.

Table 6. The TCEC14 Premier Division raw, unadjusted cross-table: three DRR phases, 42 rounds, 168 games.

\begin{tabular}{|c|c|c|c|c|c|c|c|c|c|c|c|c|c|}
\hline & ngin & LO & Pts & P\% & Elo \pm & St & Ko & Lc & Ho & Km & $\mathrm{Fi}$ & An & Et \\
\hline 1 & ish 181224 & 12 & 29.0 & 69.0 & -76 & & $=1=11$ & & $=1=1=$ & $--1-$ & $1=====$ & $111=1=$ & $1==1=1$ \\
\hline 2 & & 65 & 24.0 & 57.1 & -155 & $==0=00$ & & & & & & & $1==$ \\
\hline 3 & C2-32194 & 7 & 23.5 & 56.0 & & $===$ & $====0=$ & & $======$ & & & & \\
\hline & & & 23.5 & .0 & 01 & & $===$ & $======$ & & & $1=====$ & & $===1==$ \\
\hline & & & 18 & 42.9 & +247 & & $00==0=$ & $=-$ & & & $=1====$ & & $==1=1=$ \\
\hline 6 & & 52 & 17. & 41.7 & -148 & $0===$ & $=0=0=0$ & & $===$ & & & $======$ & $1=====$ \\
\hline 7 & & 91 & 16.5 & 39.3 & -60 & & $=====0$ & $=00$ & $=0==$ & & $=====$ & & $==1===$ \\
\hline & Ethereal 11.14 & 3377 & 16.0 & 38.1 & -48 & $0==0=0$ & $==0==$ & $===0==$ & $===0==$ & $==0=0=$ & $0=====$ & $==0===$ & \\
\hline
\end{tabular}

As in TCEC13, a knockout event was interposed between this tournament and the Superfinal. Would the LEELA team roll out an improved network in preparation for the big finish? A hint came in a 'bonus match' between a more recent 'LEELA 32585' and 'STOCKFISH 8', the latter having only 12 threads and 
a 4M hash-table. This was an echo and 'simulation' of the ALPHAZERO-STOCKFISH match: LEELA won $+24=71-5$. We report on TCEC Cup 2 separately (Haworth and Hernandez, 2019c).

Table 7. The TCEC14 Premier Division raw, unadjusted figures: head-to-head and round-by-round scores.

\begin{tabular}{|c|c|c|c|c|c|c|c|c|c|c|c|c|c|c|c|c|c|c|c|}
\hline \# & Engine & ELO & Pts & SB & St & Ko & Lc & Ho & $\mathbf{K m}$ & $\mathbf{F i}$ & An & Et & RR... & r1 & r2 & r3 & $\mathbf{r} 4$ & r5 & r6 \\
\hline 1 & ockfish 181224 & 3612 & 29.0 & 572.00 & & $4 \frac{1}{2}$ & $3 \frac{1}{1 / 2}$ & 4 & 4 & $3 \frac{1}{1 / 2}$ & 5 & $4 \frac{1}{2}$ & & $51 / 2$ & $41 / 2$ & $41 / 2$ & 5 & 5 & $4 \frac{1}{2}$ \\
\hline 2 & & & 24.0 & & $1 \frac{1}{2} 2$ & & $3 \frac{1}{1} 2$ & 3 & & $41 / 2$ & $3 \frac{1}{1} 2$ & & & 4 & $4^{1 / 2}$ & 3 & $4 \frac{1}{2}$ & 4 & \\
\hline 3 & & & & & $2^{1 / 2}$ & $2^{1 / 2}$ & & 3 & & & $41 / 2$ & & & $3 \frac{1}{1} 2$ & $4 \frac{1}{2}$ & 4 & 5 & $2^{1 / 2}$ & \\
\hline 4 & & & & & 2 & 3 & 3 & & $4 \frac{1}{2}$ & $3 \frac{1}{2} 2$ & 4 & $3 \frac{1}{2}$ & & $4 \frac{1}{2}$ & 3 & 4 & & 4 & \\
\hline 5 & $\mathrm{~K}$ & 3249 & 18 & & 2 & $1 \frac{1}{2}$ & $2^{1 / 2}$ & $1 \frac{1}{2} 2$ & & $3 \frac{1}{1} 2$ & 3 & 4 & & $2^{1 / 2}$ & $31 / 2$ & 4 & 3 & $2 \frac{1}{2}$ & $2^{1 / 2}$ \\
\hline 6 & Fire 7.1 & 3452 & 17.5 & 364.75 & $2^{1 / 2}$ & $1 \frac{1}{2}$ & 2 & $2^{1 / 2}$ & $2^{1 / 2}$ & & 3 & $3 \frac{1}{2} 2$ & & 3 & 2 & $3 \frac{1}{2} 2$ & $2^{1 / 2}$ & 4 & $21 /$ \\
\hline 7 & Andscacs & 3391 & 16.5 & 333.75 & 1 & $2^{1 / 2}$ & $1 \frac{1 / 2}{2}$ & 2 & 3 & 3 & & $3 \frac{1}{2}$ & & $2^{1 / 2}$ & $2^{1 / 2}$ & $2^{1 / 2}$ & $2^{1 / 2}$ & 3 & $3 \frac{1}{2}$ \\
\hline 8 & Ethereal 11.14 & 3377 & 16.0 & 342.00 & $1 \frac{1 / 2}{2}$ & $2^{1 / 2}$ & $2^{1 / 2}$ & $2^{1 / 2}$ & & $2 \frac{1}{2}$ & $2 \frac{1}{2}$ & & & $2^{1 / 2}$ & $3 \frac{1}{2}$ & $2^{1 / 2}$ & $1 \frac{1 / 2}{2}$ & 3 & \\
\hline
\end{tabular}

TCEC's ELOs suggested a STOCKFISH win by eleven. However, both engines came to the board in new versions: the match was now STOCKFISH v190203 versus LEELA v20.2-32930. There was bound to be a clash of styles occasioned by the different modes of evaluation and use of hardware. This dynamic was eagerly anticipated with viewer numbers often topping 2000. Jeroen Noomen (2019) again created a suitable opening book, aiming as before for at least $20 \%$ decisive results. Assaf Wool returned from his 'TCEC Cup break' to comment on all the games. GMThechesspuzzler and Kingscrusher ${ }^{3}$ were active on Youtube. Soren Riis provided the authors with detailed analysis of games 7-8, 20-22, 65-66 and 71 which we provide via our pgn file for reader convenience rather than here. GM Matthew Sadler (2019), having analysed the STOCKFISH-ALPHAZERO games (Sadler and Regan, 2019) has also contributed his own view of this Superfinal.

The play and the results did not disappoint. STOCKFISH opened its account with wins from games 7 and 10 but LEELA replied with wins from games 11 and 13. There were twelve wins in the first thirty games, a hit rate of exactly $40 \%$, see Table 8 and Fig. 2 . At this point, the score was 15-15, suggesting that this would be the closest TCEC Superfinal since Season 5 in 2013 even though LEELA had never led. The same situation appertained at 24-24 after a run of 19 draws. ${ }^{4}$ At this point, LEELA dramatically jumped out front with wins in games g49 and g53. This lead held until game 80 which STOCKFISH won. Ultimately, it was the single 0-1 win in another sea of 19 draws that allowed STOCKFISH to retain the title. Each game was closely contested with average length being one ply short of 100 moves - and not just because LEELA was reluctant to visit the draw zone.

Of course, suitably equipped grandmasters could write a book about this entirely gripping match and this would be most welcome. Here, we can only pick out a few chessic highlights which perhaps complement the analyses of the commentators above.

The hints from the evaluations of STOCKFISH suggest that it welcomed LEELA's 15. Bb2 (g07), 51.... Be3 (g08, a missed win), 34. Kf1?? (g21) and 31.... Qd6 (g22). In game 35, 29. Ke1 rather than h7 seemed to lose LEELA's winning advantage. Game 58 was adjudicated with a rare 'mate in one' on the board: the camera cut away just before the blow was struck. Game 63: LEELA was happy to trade pawns

\footnotetext{
${ }^{3}$ Wool (2019) picked out positions from games 7, 8, 10-11, 13, 16-17, 20-22, 25, 27, 29, 35, 41, 49, 53, 55, 58, 63, 65-66, 71, 75, 80, 85 and 87. Kingscrusher (2019) commentated on games 7, 10-11, 13, 16, 17, 53, 66 and 85. Games 2, 7-8, 13, 17 20, 29, 49, 65-66, 80, 85 and 100 were covered by GM Thechesspuzzler (2019).

${ }^{4}$ Not a record: the TCEC8 KomOdO 9.3x - STOCKFISH 021115 Superfinal games 14-37 and 47-71 were all draws.
} 
for position as early as eleven moves into the play. STOCKFISH did not see a serious problem until six moves later. LEELA create a passed pawn despite being three pawns down and this led to a crushing 41move win, the shortest of the match.

If there was a pivotal juncture in this Superfinal, it was games 65-66 - a crucial one or two-point swing to STOCKFISH. In game 65, LEELA missed a $\mathrm{KNP}(\mathrm{c} 4) \mathrm{P}(\mathrm{d} 5) \mathrm{KBP}(\mathrm{c} 5)$ win with the winning capture admittedly 26 moves down the line (de Man, 2018). STOCKFISH clearly saw it was lost and LEELA would have been awarded the win under the TCEC13 ' $6.5+$ ' win-adjudication rule. LEELA was within 11 ply of winning with 9 ply to go and it is worth speculating as to how soon it would have found the winning idea, $\mathrm{K}$ on b5/c6 before Nxc5, had the plycount not intervened. Game 66 had to be restarted after two server crashes before LEELA - lost. Had it been possible to return to the game-state after the last completed move, the temperature of the partisanship in the chat room would have been lower. A minor cost, but transaction-checkpoint/restart might be applicable here.

Table 8. The TCEC 14 Superfinal match of 100 games: the decisive games, Black wins underlined.

\begin{tabular}{|c|c|c|c|c|c|c|c|c|c|}
\hline Superfinal & ELO & Score & Perf. & ELO $\Delta$ & \# of & games won (0-1 wins underlined) & \# of & game-pairs won & win-pairs \\
\hline Stockfish 190203 & 3451 & $+10=81-9$ & $50.5 \%$ & -13 & $10 ?$ & $\underline{7}, 10,16,20, \underline{21}, 22,58,66,80, \underline{85}$ & 9 & $\begin{array}{l}4,5,8,10,11,29 \\
33,40,43\end{array}$ & g21-22 \\
\hline $\begin{array}{c}\text { Leela Chess Zero } \\
20.2-32930^{*}\end{array}$ & 3411 & $+9=81-10$ & $49.5 \%$ & +13 & 9 & $11,13,17,25,27,29,49,53,63$ & 9 & $\begin{array}{l}6,7,9,13,14,15, \\
25,27,32\end{array}$ & $1-0 / 0-1$ \\
\hline
\end{tabular}

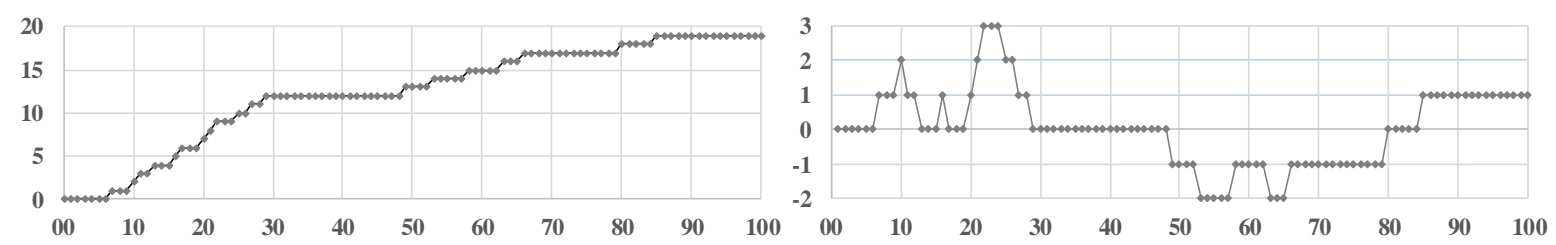

Fig. 2. The TCEC14 Superfinal's 100 games: (a) incidence of wins and (b) STOCKFISH's net score.

Game 85 was the final win: the 12-move King's Indian opening had already defined the major asymmetry of Queen versus BBPP. LEELA went from apparent equality to negative territory by move 25. Ultimately, LEELA's QR were unable to prevent mate by a BBNNPP team, only five moves away when the referee stepped in. Game 86 was the longest ever TCEC game at 362 moves.

\section{The Bonus 4-way and 2-way Rapid events}

TCEC treated us to two bonus event at the Rapid tempo of $12^{\prime}+3^{\prime \prime} /$ move. The first featured the top four - HOUDINI, KOMODO, LEELA CHESS ZERO and STOCKFISH: 20 DRRs, 40 round robins, 120 rounds and 240 games. STOCKFISH had a good first half and was never headed even if pursued closely by LEELA. HOUDINI and KOMODO tailed off, eventually in that order as KOMODO fared poorly in the second half. ELO-predicted net scores were $+9 /+1 /-2 /-8$ but 'actuals' were $+12 /+6 /-7 /-11$. The longest wins were g116.1 (1-0, 139 moves) and g37.2 (0-1, 125m): the longest draw, g12.1 (318m). Game 18.1 between Leela and Komodo was something of an anti-climax as a 3x-repetition draw after ten played moves. Full details are included with the repository e-version of this note (Haworth and Hernandez, 2019d).

The second event was a 100-game STOCKFISH-LEELA match from the initial position: no prescribed openings. LEELA won 16-4, perhaps by being single-minded about its openings (Wool, 2019). 


\section{Summary}

The Google DeepMind company in St. Pancras, London have been remarkably open in sharing the core ideas of their intelligence initiative. In the year it has taken for DeepMind's papers on ALPHAZERO (Silver et al, 2017, 2018) to mature and satisfy the referees, we have seen TCEC invest in Nvidia GPUs and foster several innovations going beyond the classic Shannon (1950) minimaxing AB model of a chess engine. We have seen a leading chess-engine author, Mark Lefler, move his focus successfully from top engine KOMODO to KOMODO MCTS (Chessdom, 2018). With one less technical break, this engine would have come all the way through the divisions to fully justify its place in Division $\mathrm{P}$ at the first attempt.

We have also seen a community come together to support and train the open-source LEELA CHESS ZERO echo of ALPHAZERO. Again, this has been rewarded by success, and how. LEELA edged out KOMODO and HOUDINI to take the challenger's place in the Superfinal here. It was not expected to beat STOCKFISH but came within one game of drawing the classic phase.

Table 9. Generic statistics for each phase of TCEC14: results, terminations and average game-length.

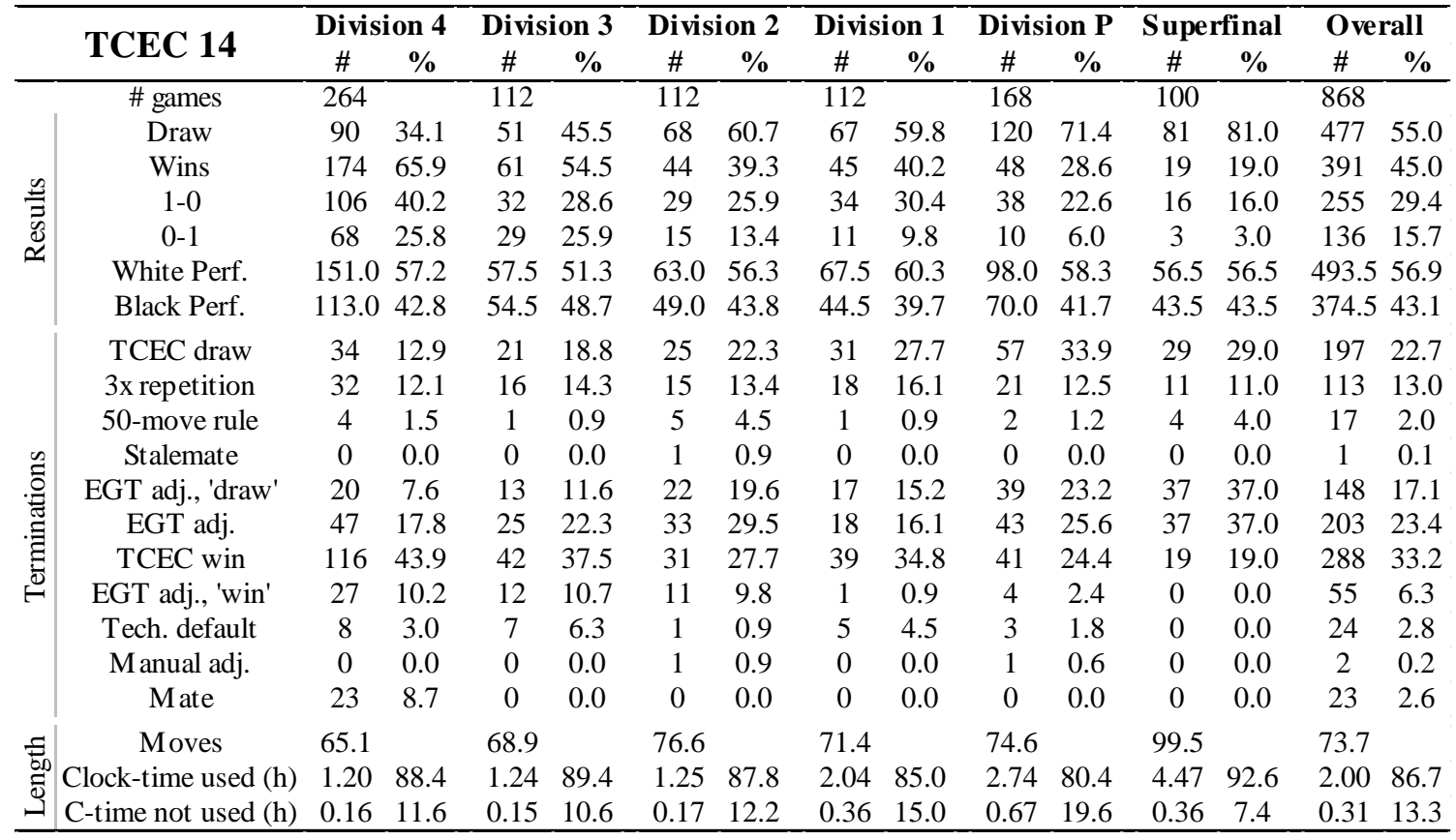

Table 10. The shortest and longest 1-0, drawn and 0-1 games in each phase of TCEC14.

\begin{tabular}{|c|c|c|c|c|c|c|c|c|c|c|c|c|c|c|c|c|c|c|}
\hline \multirow{3}{*}{ Div. } & \multicolumn{6}{|c|}{$1-0$} & \multicolumn{6}{|c|}{$1 / 2-1 / 2$} & \multicolumn{6}{|c|}{$0-1$} \\
\hline & \multicolumn{3}{|c|}{ Shortest } & \multicolumn{3}{|c|}{ Longest } & \multicolumn{3}{|c|}{ Shortest } & \multicolumn{3}{|c|}{ Longest } & \multicolumn{3}{|c|}{ Shortest } & \multicolumn{3}{|c|}{ Longest } \\
\hline & $1.4 / 4$ & $\mathrm{Ne}-\mathrm{Sn}$ & 30 & $41.1 / 241$ & Tu-Wi & 126 & $40.1 / 235$ & Wi-Ne & 18 & $37.3 / 219$ & ro-Tu & 183 & $9.5 / 53$ & Sn-c22 & 29 & $36.2 / 212$ & c22-Km & 127 \\
\hline 2 & $1.1 / 1$ & $\mathrm{Km}-\mathrm{Ni}$ & 33 & $12.3 / 47$ & $\mathrm{Km}-\mathrm{Cb}$ & 139 & $17.3 / 67$ & $\mathrm{Xi}-\mathrm{Te}$ & 22 & $25.4 / 100$ & Bo-Lc & 216 & $21.3 / 83$ & Gu-Lc & 50 & $19.2 / 74$ & Xi-Lc & 139 \\
\hline 1 & $3.4 / 12$ & La-Fr & 43 & $28.3 / 111$ & Lc-Fr & 308 & $18.2 / 70$ & Jo-La & 26 & $11.3 / 43$ & Gi-Km & 163 & $25.3 / 99$ & Gi-Km & 22 & $22.3 / 87$ & Fr-Ch & 103 \\
\hline $\mathbf{P}$ & $1.3 / 3$ & Ho-An & 45 & $12.4 / 48$ & Lc-An & 128 & $36.2 / 142$ & Lc-Ko & 28 & $17.3 / 67$ & Fi-Lc & 209 & $40.1 / 157$ & $\mathrm{Km}-\mathrm{Ho}$ & 56 & $37.4 / 148$ & Ko-St & 112 \\
\hline $\mathbf{F}$ & 63 & Lc-St & 41 & 53 & Lc-St & 111 & 59 & Lc-St & 31 & 86 & St-Lc & 362 & 21 & Lc-St & 49 & 85 & Lc-St & 64 \\
\hline
\end{tabular}


Chess 24 and Chessbomb, with its useful colour-coding of moves, covered the TCEC14 Superfinal so we were treated to kibitzing by three different, objective but hardly neutral versions of STOCKFISH. The Twitch TCEC channel claims that viewers' computers have to date had a window open to TCEC Seasons 10-14 for a total of over half a million hours.

\section{REFERENCES}

CPW (2019). https://tinyurl.com/icga046. The Chess Programming Wiki website, including biographies of engines, authors and developers.

Chessdom (2018). http://tinyurl.com/icgak034. Interview with Mark Lefler and Larry Kaufman.

de Man, R. (2018). http://tablebase.sesse.net/syzygy/. Site providing sub-8-man DTZ 50 " EGTs.

'GM Thechesspuzzler' (2019). https://tinyurl.com/icga056. Superfinal video-commentaries.

Haworth, G. McC. and Hernandez, N. (2019a). http://centaur.reading.ac.uk/80284/. TCEC Cup 1. ICGA Journal, Vol. 41(1) 31-38. doi:10.3233/ICG-190099.

Haworth, G. $M^{\mathrm{c}}$ C. and Hernandez, N. (2019b). http://centaur.reading.ac.uk/78820/. TCEC13: the $13^{\text {th }}$ Top Chess Engine Championship. ICGA Journal, Vol. 41(2) 92-99. doi:10.3233/ICG-190103.

Haworth, G. $\mathrm{M}^{\mathrm{c}} \mathrm{C}$. and Hernandez, N. (2019c). http://centaur.reading.ac.uk/81390/. TCEC Cup 2. ICGA Journal, Vol. 41(2) 100-107. doi:10.3233/ICG-190104.

Haworth, G. $\mathrm{M}^{\mathrm{c}} \mathrm{C}$. and Hernandez, N. (2019d). http://centaur.reading.ac.uk/82052/. TCEC14: this note plus annotated pgn files and statistics. ICGA Journal, Vol. 41(3) 143-151. doi:10.3233/ICG-190117.

Intel (2017) https://tinyurl.com/icga042. Intel's specification of the $\mathrm{XEON}^{\circledR} \mathrm{E}^{\mathrm{E}-2699 \mathrm{~V} 4}$ processor.

Kingscrusher (2019). http://tinyurl.com/icgaj057. Superfinal video commentaries.

Noomen, J. (2019). https://tinyurl.com/icgaj054. JN's approach to the Superfinal openings.

Nvidia (2018). https://www.nvidia.com/en-gb/geforce/graphics-cards/rtx-2080-ti/ GEFORCE GTX 2080 TI GPU specification and benchmark performance data.

Romero, P. P. (2012) https://tinyurl.com/icga013. FINALGEN: tutorial, download and forum.

Sadler, M. (2019). The TCEC14 Computer Chess Superfinal: a perspective. ICGA Journal, 41(3) 152. doi:10.3233/ICG-190116. See also https://tinyurl.com/icga055.

Sadler, M. and Regan, N. (2019). Game Changer: AlphaZero's Groundbreaking Chess Strategies and the Promise of AI. New in Chess. ISBN 978-90-5691-818-7.

Shannon, C. E. (1950). Programming a Computer for Playing Chess. The London, Edinburgh and Dublin Philosophical Magazine, 41(314), 256-275. https://doi.org/10.1080/14786445008521796.

Silver, D. et al (2017). Mastering Chess and Shogi by Self-Play with a General Reinforcement Learning Algorithm. arXiv: 1712.01815.

Silver, D. et al (2018). A general reinforcement learning algorithm that masters chess, shogi, and Go through self-play. Science, 362(6419), 1140-4. doi:10.1126/science.aar6404.

Wool, A. (2019) http://mytcecexperience.blogspot.co.uk/ TCEC blog. 